\title{
Description et cycle de Ecbinoparypbium combesi sp. n. chez Bulinus truncatus
}

vecteur de Schistosoma baematobiumi en Algérie

\author{
par N. KECHEMIR \\ (Collaboration technique : M. TouAMI)
}

Service d'Epidémiologie (Directeur : Pr O. Tabet-Derraz), Institut Pasteur d'Algérie, Rue $D^{\prime}$-Laveran, Alger, Algérie.

\section{Résumé.}

L'adulte, les stades de développement ainsi que le cycle évolutif de Echinoparyphium combesi sp. n., trouvé en Algérie, sont décrits. Les sporocystes et rédies se développent chez Bulinus truncatus. Dans la nature, les cercaires émises par les Bulins s'enkystent dans la cavité péricardique de Physa acuta et de B. truncatus lui-même. Expérimentalement, elles peuvent s'enkyster chez Planorbarius metidjensis et également dans les reins de têtards de Rana ridibunda perezi. Elles peuvent aussi s'enkyster sur place dans le $1^{\text {er }}$ hôte intermédiaire. L'évolution de la métacercaire en adulte se fait expérimentalement dans l'intestin grêle du poussin domestique et de la souris blanche de laboratoire.

Le parasite provoque la castration du mollusque d'où son intérêt pour une éventuelle intervention sur les populations de Bulins vecteurs de Schistosoma haematobium.

\section{Summary.}

Description and life cycle of Echinoparyphium combesi sp.n. in Bulinus truncatus, vector of Schistosoma haematobium in Algeria.

The adult worm, developmental stages and life cycle of Echinoparyphium combesi, found in Algeria, are described. Sporocysts and rediae develop in Bulinus truncatus. In natural conditions cercariae released by the snail Bulinus encyst in the pericardial cavity of Physa actua and Bulinus truncatus itself. Experimentally they may encyst in Planorbarius metidjensis and also in the kidneys of Rana ridibunda perezi tadpoles. They can also encyst

Accepté le 21 septembre 1979. 
in situ in the first intermediate host. Experimentally, metacercariae mature in the small intestine of chicks and mice. The parasite provokes castration in the genital gland of the snail. It could therefore be used in biological control of Bulinus, the vector of Schistosoma haematobium.

Au cours de l'étude sur la biologie de B. truncatus (1), hôte intermédiaire de $S$. haematobium en Algérie, nous avons rencontré des stades larvaires appartenant à un Trématode Echinostomatidae du genre Echinoparyphium. Les Bulins ont été récoltés dans une mare de la banlieue d'Alger (El-Harrach) récemment identifiée comme site de transmission de la bilharziose urinaire (Tabet-Derraz, Belkaid, Naceur, Ouchfoun, 1977).

Nous avons réalisé expérimentalement le cycle de ce trématode dans le but de déterminer son influence éventuelle sur la dynamique des populations de Bulins.

\section{Adulte (fig. $1 \mathrm{~A})$}

\section{DESCRIPTION :}

Les mensurations de 6 individus obtenus expérimentalement chez le poussin sont les suivantes :

longueur du corps : 3394 à $4174 \mu \mathrm{m}(3$ 810);

largeur du corps : 610 à $840 \mu \mathrm{m}$ (742) ;

ventouse orale : 102 à $132 / 127$ à $150 \mu \mathrm{m}(118 / 138)$;

ventouse ventrale : 435 à $460 / 370$ à $452 \mu \mathrm{m}(440 / 417)$;

rapport ventousaire moyen : 0,29 ;

pharynx : 102 à $150 / 88$ à $98 \mu \mathrm{m}(116 / 90)$;

ovaire : 180 à 225/180 à $212 \mu \mathrm{m}(205 / 191)$;

testicule antérieur : 430 à 630/200 à $285 \mu \mathrm{m}(519 / 250)$;

testicule postérieur : 530 à $700 / 165$ à $255 \mu \mathrm{m}(580 / 220)$.

Le corps allongé, à extrémité antérieure pourvue d'un collet réniforme (321 à $353 \mu \mathrm{m} / 143$ à $184 \mu \mathrm{m}$ ) est légèrement effilé dans sa région postérieure. Dans la zone pré-acétabulaire il est aplati avec une concavité ventrale.

Le collet (fig. 2) est muni de 43 épines d'inégales dimensions qui forment un collier interrompu ventralement dans la dépression médiane. Les épines dorsales sont au nombre de 27 et disposées en 2 rangs ; on observe 14 petites (28 à $45 \mu \mathrm{m} / 10$ à $17 \mu \mathrm{m}$ ) alternant avec 13 grandes (34 à $66 \mu \mathrm{m} / 12,5$ à $19,5 \mu \mathrm{m}$ ) qui sont légèrement plus postérieures. Les épines ventrales sont au nombre de 16 réparties en 2 lots

(1) Nous adressons nos remerciements à $\mathrm{M}$. le $\mathrm{P}^{\mathrm{r}} \mathrm{G}$. Mandahl-Barth, qui a bien voulu nous déterminer les Mollusques. 


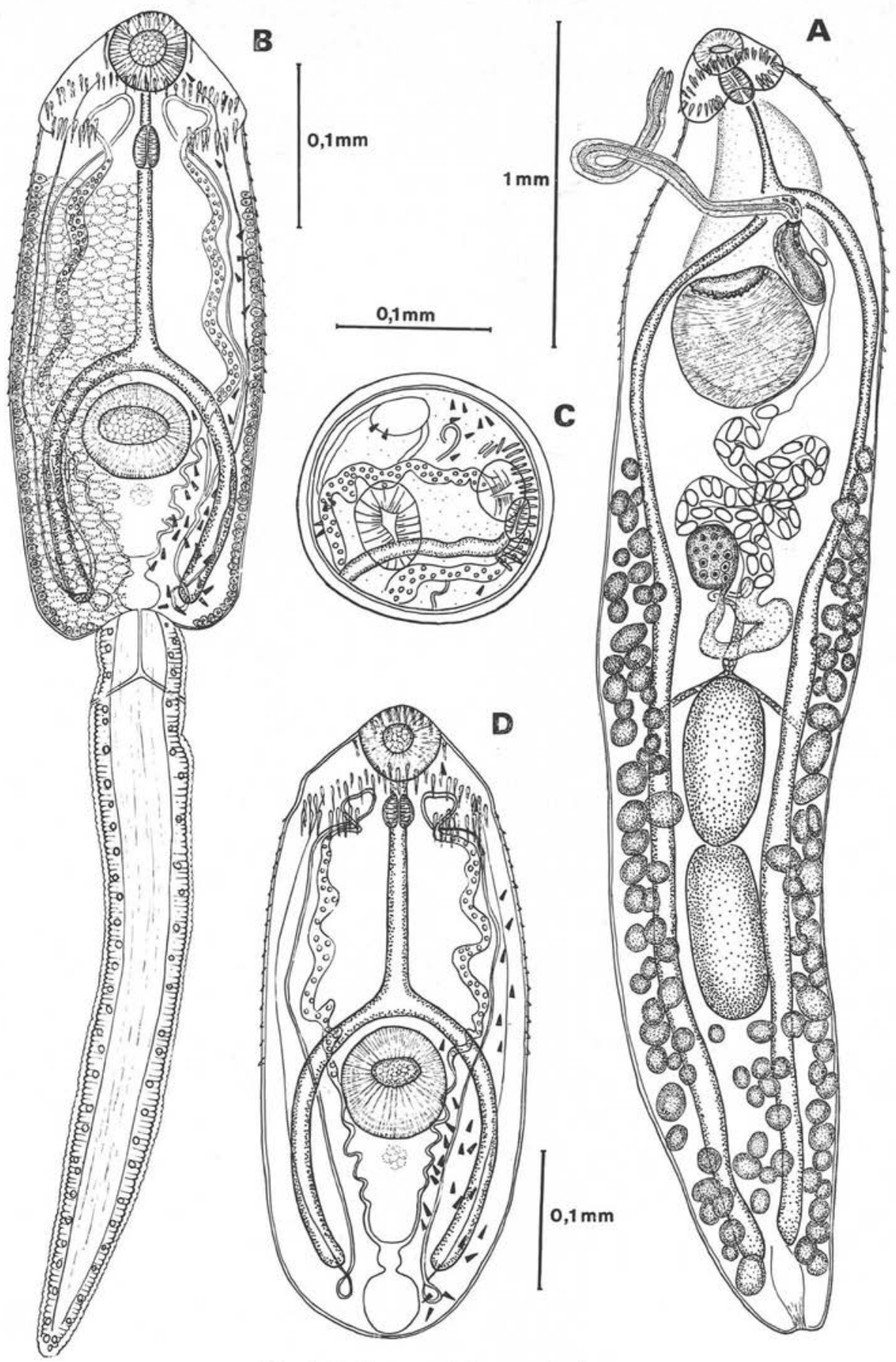

Fig. 1. Echinoparyphium combesi sp.n.

A : adulte, face ventrale ; B : cercaire ; C : métacercaire enkystée ; D : métacercaire extraite du kyste. 
symétriques de part et d'autre de la dépression du collet; chaque lot comprend : 5 épines (51 à $64,5 \mu \mathrm{m} / 14$ à $19,5 \mu \mathrm{m}$ ) groupées et implantées sur 3 niveaux et 3 autres un peu plus petites $(43$ à $61 \mu \mathrm{m} / 11$ à $16 \mu \mathrm{m})$ situées sur une même rangée. Buscher (1978) propose une formule pour traduire la disposition des épines du collet des Echinoparyphium; si on l'applique à nos exemplaires l'arrangement de leurs épines peut se formuler de la façon suivante : 5-5-3-3-27 (14-13).

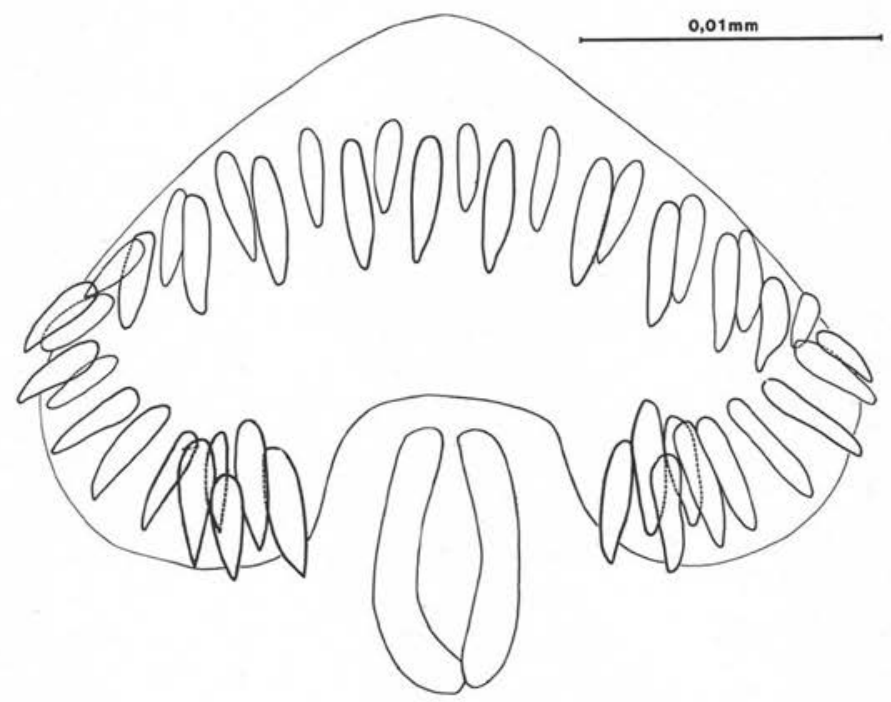

Fig. 2. Echinoparyphium combesi sp.n.

Disposition des épines du collet, vue ventrale.

Le tégument présente une spinulation antérieure qui s'étend dorsalement jusqu'au niveau de la bifurcation caecale et ventralement jusqu'au niveau du deuxième tiers de l'acétabulum.

La ventouse orale est terminale. La ventouse ventrale, volumineuse, est située dans le tiers antérieur du corps et présente une ouverture dentelée.

La bouche s'ouvre sur un prépharynx visible uniquement sur quelques spécimens; au pharynx ovale et musculeux fait suite un long œsophage qui se bifurque en 2 cæcums étroits qui longent les bords du corps jusqu'au niveau des vitellogènes ; ensuite ces derniers les séparent du bord jusqu'à la fin de leur parcours. Les cæcums n'atteignent pas l'extrémité postérieure du ver et sont en retrait par rapport aux derniers follicules vitellins.

Les deux testicules localisés dans le troisième quart de l'animal sont dans l'axe médian du corps. Ils ont une forme allongée, arrondie aux extrémités et sont contigus. Le testicule antérieur est plus petit que le postérieur. La poche du cirre est située à gauche de l'acétabulum et ne dépasse pas vers l'arrière le tiers antérieur de celui-ci ; 
elle contient une vésicule séminale assez volumineuse suivie d'une pars prostatica et d'un cirre très long, inerme, à extrémité granuleuse.

L'ovaire sphérique est situé entre l'acétabulum et le testicule antérieur. Il est légèrement décalé à droite de l'axe du corps. De l'oviducte cilié, précédé d'un oocapte conique, part un court canal de Laurer qui s'ouvre dorsalement. Les vitellogènes, constitués par de gros follicules, s'étendent du bord postérieur de la ventouse ventrale jusqu'à l'extrémité du corps ; jusqu'au bord inférieur du testicule postérieur ils sont latéraux ; dans la zone post-testiculaire on observe des follicules intercæcaux. Les vitelloductes transverses situés en avant du testicule antérieur se rejoignent pour donner un vitelloducte impair, jouant le rôle de réservoir vitellin. L'utérus débute par une dilatation servant de réceptacle séminal ; il est court et contient de 36 à 58 œufs. Le pore génital commun se trouve en avant de l'acétabulum, au niveau de la bifurcation cæcale.

La vessie excrétrice est en forme d'ampoule, son pore excréteur se situe à l'extrémité postérieure du corps.

Les œufs, de forme ovale, sont de couleur ocre. Dans l'utérus, ils mesurent de 85 à $97 \mu \mathrm{m}$ de long sur 45 à $58 \mu \mathrm{m}$ de large. Ils sont pondus non embryonnés. Au bout de 10 jours à $28^{\circ}$, un miracidium animé de légers mouvements est visible à l'intérieur de la coque operculée.

\section{Discussion.}

Par l'ensemble de ses caractères (notamment l'utérus court, la forme du collier d'épines et les testicules régulièrement ovales) notre Trématode prend place dans le genre Echinoparyphium.

Dans un travail récent, Buscher (1978) a établi une clé de détermination des espèces du genre Echinoparyphium. Il a supprimé du genre un bon nombre d'espèces qui étaient retenues par Skrjabin (1956) et par Yamaguti (1971); parmi celles qu'il prend en considération, 6 seulement ont un collier de 43 épines; il s'agit de : E. elegans (Loss, 1899), Dietz, 1909, E. bioccalerouxi Dollfus, 1953, E. dunni Lie et Umathevy, 1965, E. paracinctum Bychovskaya-Pavlovskaya, 1953, E. ralphaudyi Lie et al., 1975 et E. syrdariense Burdelev, 1937. Si nous nous référons à cette clé, fondée sur le nombre et l'arrangement des épines et l'éventualité du réceptacle séminal et de la pars prostatica, notre espèce correspondrait à l'espèce égyptienne ralphaudyi; or en prenant d'autres caractères que ceux utilisés par Buscher, nous avons mis en évidence plusieurs différences entre nos exemplaires algériens et $E$. ralphaudyi :

1. la poche du cirre n'atteint jamais le bord postérieur de l'acétabulum et ne dépasse même pas la moitié antérieure de celui-ci alors que chez E. ralphaudyi elle arrive au bord postérieur;

2. le cirre est plus long chez nos exemplaires; les deux mesures que nous avons prises $(1167$ et $1015 \mu \mathrm{m})$ sont supérieures à celle donnée $(800 \mu \mathrm{m})$ pour ralphaudyi; 
3. les follicules vitellins sont toujours confluents en arrière des testicules alors que la zone intercæcale en est dépourvue chez l'espèce ralphaudyi;

4. les testicules n'ont pas les mêmes dimensions : 300 à $590 \mu \mathrm{m} / 120$ à $360 \mu \mathrm{m}$ chez ralphaudyi; 430 à $630 \mu \mathrm{m} / 200$ à $285 \mu \mathrm{m}$ et 530 à $700 \mu \mathrm{m} / 165$ à $255 \mu \mathrm{m}$ pour notre espèce ;

5. les œufs paraissent moins nombreux (36 à 58) que chez ralphaudyi (15 à 90).

En dehors des espèces retenues par Buscher dans le genre Echinoparyphium, nous avons comparé nos exemplaires à l'ensemble des espèces validées par Skrjabin et Yamaguti sans pouvoir les identifier à aucune d'entre elles.

En conséquence, nous considérons que nos exemplaires appartiennent à une espèce nouvelle, que nous dédions à $\mathrm{M}$. le $\mathrm{P}^{\mathrm{r}} \mathrm{C}$. Combes et pour laquelle nous proposons le nom de Echinoparyphium combesi $\mathrm{sp}$. n. Il s'agit de la première espèce du genre en Algérie.

\section{Stades larvaires}

RÉDIE.

Les mensurations d'une dizaine de rédies filles rencontrées dans un Bulin parasité naturellement sont les suivantes :

longueur du corps : 460 à $1720 \mu \mathrm{m}(1051)$;

largeur du corps : 160 à $226 \mu \mathrm{m}(220)$;

pharynx : 39,5 à $118 \mu \mathrm{m} / 42,5$ à $72,5 \mu \mathrm{m}(72,5 / 67)$.

La rédie, de forme allongée, est colorée en orange. Le pharynx est suivi d'un court cæcum digestif ( $74 \mu \mathrm{m}$ environ) au niveau duquel se trouve un collier musculeux. Il existe deux protubérances un peu en avant du tiers postérieur de la rédie. Le nombre de cercaires contenues dans une rédie varie de 6 à 11 .

Cercaire (fig. 1, B).

Les dimensions de 10 spécimens fixés à froid par une solution de formol à $5 \%$ sont les suivantes :

longueur du corps : 780 à $897 \mu \mathrm{m}(878)$;

largeur du corps : 143 à $184 \mu \mathrm{m}(157)$;

ventouse orale : 46 à $55 \mu \mathrm{m} / 42$ à $50 \mu \mathrm{m}(50 / 48)$;

ventouse ventrale : 60 à $69 / 55$ à $72 \mu \mathrm{m}(65 / 61)$;

rapport ventousaire moyen : 0,77 ;

pharynx : 27 à $32 / 19$ à $21 \mu \mathrm{m}(29 / 20)$.

Le corps est allongé, à extrémité antérieure légèrement triangulaire et munie d'un collet garni d'une couronne de 43 épines. L'extrémité postérieure est arrondie. Le tégument de la zone préacétabulaire est pourvu dorsalement et ventralement d'une 
fine spinulation. La ventouse orale est terminale, la ventrale conique à ouverture circulaire se trouve dans la moitié postérieure du corps.

La bouche s'ouvre sur un prépharynx, suivi d'un pharynx et d'un long œsophage. Ce dernier se bifurque juste avant l'acétabulum pour donner deux cæcums arqués qui n'atteignent pas l'extrémité postérieure de l'animal.

A la pointe antérieure du corps, nous avons pu observer huit orifices qui correspondent aux ouvertures des canaux des cellules glandulaires; deux autres se trouvent de part et d'autre de la ventouse orale. A la surface interne du tégument, sauf en avant du pharynx, se trouvent des cellules cystogènes granuleuses.

L'appareil excréteur est parfaitement visible dans la partie située entre le pharynx et l'acétabulum grâce à la présence dans les collecteurs principaux de gros granules réfringents.

Au niveau de l'acétabulum, ces collecteurs regagnent la vessie par un canal tortueux. Dans la zone pré-pharyngienne, où ils deviennent moins épais, ils forment une boucle et redescendent à l'extrémité postérieure de l'animal. Nous avons dénombré 30 à 32 paires de cellules flammes ( $24 \mathrm{chez}$ E. ralphaudyi). La vessie, à pôle antérieur renflé en petite ampoule, envoie dans la queue un fin canalicule qui se dichotomise ; chacun des segments est terminé par un pore excréteur.

Contrairement aux observations de Lie et Umathevy (1965) et Lie et al. (1975) pour E. dunni et E. ralphaudyi, l'ébauche de l'appareil génital se limite chez nos exemplaires à une unique masse cellulaire, à peine perceptible, dans la zone postacétabulaire.

\section{Métacercaire ( $f$ ig. $1 \mathrm{C}$ et $\mathrm{D}$ ).}

La métacercaire est contenue dans un kyste sphérique ou légèrement ovale ayant pour dimensions 150 à $172 \mu \mathrm{m}$ sur 150 à $190 \mu \mathrm{m}$. La paroi du kyste est constituée par deux enveloppes; la plus externe, transparente, a une épaisseur variant de 8 à $10 \mu \mathrm{m}$, l'autre, plus mince, a une épaisseur variant de 5 à $6 \mu \mathrm{m}$.

Nous donnons ci-après les mensurations de huit individus dékystés obtenus à partir de Bulins parasités naturellement :

longueur du corps : 300 à $470 \mu \mathrm{m}(383)$;

largeur du corps : 92 à $150 \mu \mathrm{m}(125)$;

ventouse orale : 44 à $52 / 42$ à $54 \mu \mathrm{m}(48 / 47)$;

ventouse ventrale: 50 à $80 / 47$ à $72 \mu \mathrm{m}(64 / 63)$;

rapport ventousaire moyen : 0,74 ;

pharynx : 25 à $32 / 22$ à $29 \mu \mathrm{m}(30 / 25)$.

La métacercaire extraite de son kyste présente une forme qui rappelle celle de la cercaire; par rapport à cette dernière, elle a un acétabulum et une bifurcation cæcale qui occupent une position plus centrale, la forme des cæcums digestifs et l'aspect de l'appareil excréteur restent les mêmes, l'ébauche de l'appareil génital n'est pas plus marquée. 


\section{Mode de transmission}

DONNÉES NATURELLES.

Dans le biotope naturel, qui est une mare permanente, les cercaires d'E. combesi sp. n. sont émises par le Gastéropode Pulmoné, Bulinidae: Bulinus truncatus (Audouin).

Nous avons prélevé entre le 30 janvier 1978 et le 12 juin 1978, à raison d'une récolte par mois, 554 Mollusques : 66 étaient positifs; la prévalence est donc de $12 \%$ en moyenne.

Les métacercaires enkystées sont hébergées soit par l'espèce Bulinus truncatus elle-même, soit par un autre Gastéropode Pulmoné, Physidae: Physa acuta (Drap.). Elles sont généralement rassemblées sous forme de grappe dans la région péricardique du Mollusque, mais se rencontrent aussi au niveau du rein ou même dans tous les organes chez les Bulins porteurs de rédies émettant des cercaires; dans ce cas elles correspondent probablement à des cercaires qui n’ont jamais quitté le premier hôte intermédiaire et se sont enkystées sur place. Moravec et al. (1974) ont fait la même observation chez E. recurvatum; ils signalent en plus des métacercaires enkystées à l'intérieur des rédies. Pour E. dunni et E. ralphaudyi Lie et Umathévy (1965) et Lie et al. (1975) mentionnent également cette localisation de la métacercaire. En ce qui nous concerne, nous n'avons jamais rencontré de rédie contenant ce stade enkysté.

Nous n'avons pas découvert l'hôte définitif naturel de E. combesi sp. n.

\section{DONNÉES EXPÉRIMENTALES.}

Infestation du deuxième hôte intermédiaire.

Expérimentalement, nous avons pu obtenir l'enkystement des métacercaires chez trois Gastéropodes Pulmonés: Physa acuta (Drap.), Bulinus truncatus (Aud.) et Planorbarius metidjensis (Forbes), tous élevés au laboratoire. Dans le cas de Bulinus truncatus, nous n'avons jamais trouvé, comme dans les infestations naturelles, de métacercaires situées ailleurs que dans la région péricardique. Avec Planorbis planorbis (L.), nos essais d'infestation se sont soldés par un échec. Par contre, nous avons également obtenu des résultats positifs en infestant des têtards d'Amphibiens appartenant à l'espèce Rana ridibunda perezi (Seoane, 1885) ; les cercaires, comme l'ont observé Richard et Brygoo (1978) pour Echinostoma caproni, pénètrent par l'orifice cloacal et vont se loger dans les reins de leur hôte.

\section{Infestation de l'hôte définitif.}

Les espèces du genre Echinoparyphium étant à l'état adulte parasites d'Oiseaux et de Mammifères (l'homme y compris d'après la récente, note de Fain et Galal, 1977), nous avons choisi comme hôtes définitifs expérimentaux le poussin domestique et la souris blanche de laboratoire. 
Nous avons fait ingérer à ces deux hôtes des métacercaires enkystées obtenues expérimentalement ou prélevées dans des Mollusques parasités naturellement; dans tous les cas, l'incision de l'intestin nous a permis de recueillir des juvéniles et des adultes en arrière de la région duodénale. Les individus mûrs ont été récoltés à partir du $20^{\mathrm{c}}$ jour après l'infestation.

\section{Discussion.}

Nos données naturelles et expérimentales nous permettent de conclure que nous sommes en présence d'un cycle évolutif présentant simultanément deux modes possibles (fig. 3) :

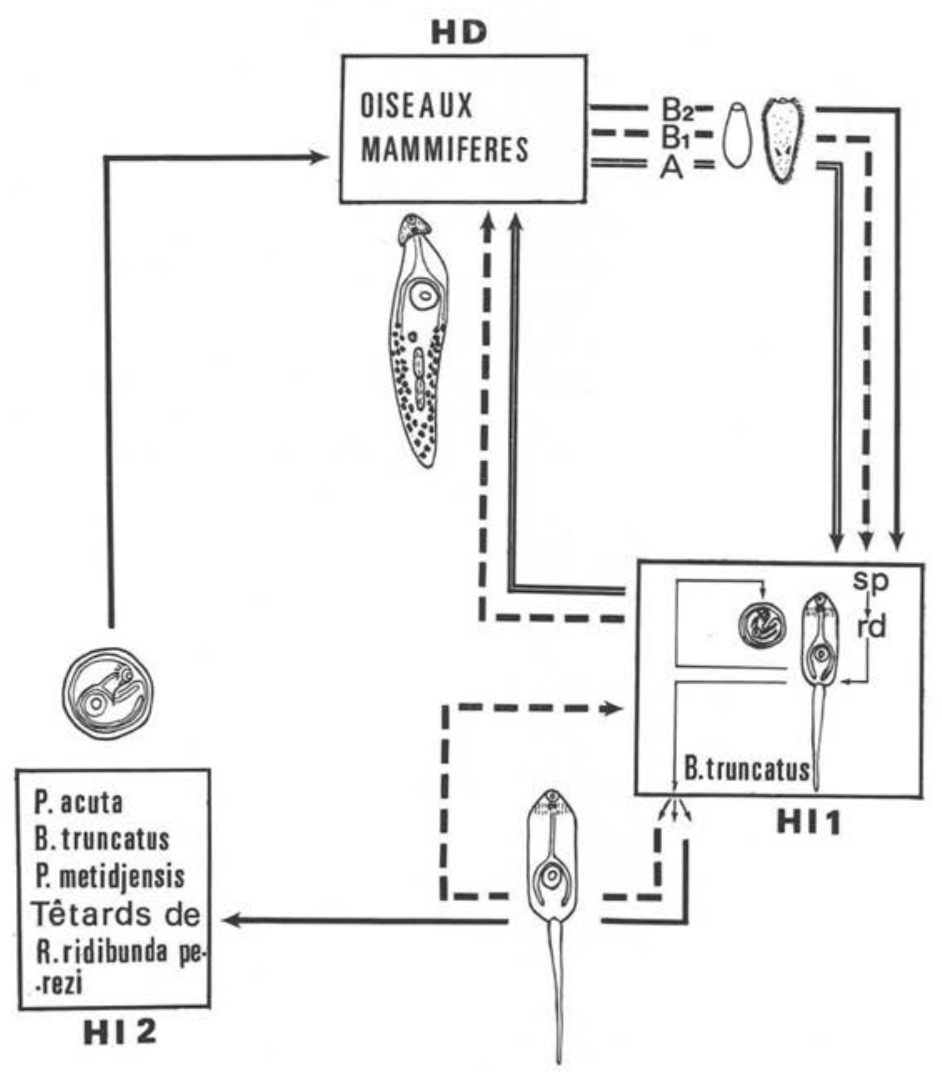

Fig. 3. Cycle évolutif d'Echinoparyphium combesi sp.n.

$\mathrm{A}$ : mode à deux hôtes sans dispersion cercarienne; $\mathrm{B}_{1}$ et $\mathrm{B}_{2}$ : mode à trois hôtes avec dispersion cercarienne; $\mathrm{B}_{1}$ : cycle avec possibilité pour le même Bulin de servir de $1^{\text {er }}$ hôte intermédiaire et de $2^{e}$ hôte intermédiaire $; \mathrm{B}_{2}$ : cycle avec un $2^{\circ}$ hôte intermédiaire pouvant être une Physe, un Planorbe, un autre Bulin ou un têtard de grenouille. 
1. un mode à deux hôtes sans dispersion cercarienne, le Bulin héberge des métacercaires enkystées dans un organe quelconque du Mollusque; elles correspondent à des cercaires qui n'ont jamais été émises à l'extérieur du Mollusque,

2. un mode à trois hôtes avec obligatoirement une dispersion cercarienne. Le premier hôte intermédiaire est toujours le Bulin. Le deuxième hôte intermédiaire peut être une Physe, un Planorbe, un têtard ou à nouveau un Bulin; dans ce deuxième hôte intermédiaire la localisation des métacercaires est toujours limitée à la région péricardique de l'organisme. Dans le cas de Bulinus truncatus précisément, des cercaires émises par un individu donné peuvent revenir s'enkyster dans ce même individu; leur enkystement focalisé dans la zone péricardique permet de distinguer ce cas du mode précédent.

\section{Effet stérilisant}

Des coupes sériées, de 8 à $9 \mu \mathrm{m}$ d'épaisseur, d'individus parasités naturellement ont révélé l'absence d'ovotestis à structures acineuses entières; nous avons seulement pu déceler, entre les métacercaires enkystées du parasite, quelques reliquats d'acini et des têtes spermatiques dans le canal hermaphrodite. Ce type de castration parasitaire par les stades larvaires de Trématodes a été envisagé comme une voie possible de recherche en vue d'un contrôle biologique des vecteurs de schistosomoses (Huizinga, 1973, Golvan et al., 1975, Combes, 1976 et Nassi, 1978).

Dans le cas de $E$. combesi sp. n., trois éléments nous paraissent $a$ priori très favorables :

- les métacercaires exercent une action pathologique complémentaire de celle des parthenitae ;

- le cycle peut se dérouler avec deux hôtes seulement;

- la souris de laboratoire est un hôte réceptif, ce qui pourrait permettre une production en masse d'œufs relativement aisée.

Nous comptons dans un premier temps rechercher l'impact naturel qu'E. combesi sp. n. exerce sur les populations de Bulins dans les sites de transmission de Schistosoma haematobium.

\section{Conclusion}

Echinoparyphium combesi sp. n. utilise comme premier hôte intermédiaire Bulinus truncatus vecteur de Schistosoma haematobium, d'où l'intérêt que peut présenter son étude. Il est à noter que le genre Echinoparyphium est signalé pour la première fois en Algérie. 
E. Combesi, par la destruction de la gonade qu'il provoque, stérilise le Bulin, il peut de ce fait prendre une part importante dans sa démographie et son utilisation dans un contrôle biologique de $S$. haematobium pourrait être envisagée.

En plus des phénomènes de castration, lors d'infestations mixtes Echinostomatidae-Schistosomatidae, il a été démontré par divers auteurs, Moravec et al. (1974 a et b), Rysavy et al. (1974), Lie et al. (1968), Heyneman et al. (1972), Lie (1973), Basch et Diconza (1975), qu'il existait un antagonisme entre les espèces. Cet antagonisme entraîne chez le gastéropode vecteur la prédominance de l'Echinostomatidae ; cela fait une deuxième raison d'envisager l'utilisation de ces Trématodes dans la lutte biologique contre $S$. haematobium.

\section{Bibliographie}

Basch P. F., Diconza J. J. (1975) : Predation by Echinostome rediae upon Schistosome sporocysts in vitro. J. Parasitol., 61, 1044-1047.

Bisseru B. (1967): Stages in the development of larval Echinostomes recovered from Schistosome transmitting Mollusc in central Africa. J. Helm., 41, 89-108.

Buscher H.N. (1978) : Echinoparyphium speotyto sp. n. (Trematoda : Echinostomidae) from the burrowing owl in Oklahoma, with a discussion of the genus Echinoparyphium. J. Parasitol., 64, $52-58$.

Combes C. (1976): Perspectives actuelles d'utilisation des parasites dans la lutte contre les Mollusques vecteurs des bilharzioses humaines. Wiadomosci Parazytol., 22, 351-354.

Fain A., Galal A. (1977) : Présence du Trématode Echinoparyphium recurvatum (von Linstow, 1873) chez l'homme et chez un rongeur d'Egypte. Ann. Soc. Belge Med. Trop., 57, 583-587.

Golvan Y.-J., Combes C., Nassi H. (1975): Castration du Mollusque Biomphalaria glabrata par les larves de divers Trématodes guadeloupéens. C.R. Acad. Sci. Paris, 280, (série D), 1607-1610.

Heyneman D., Lim H. K., Jeyarasingam V. (1972) : Antagonism of Echinostoma liei (Trematoda : Echinostomatidae) against the Trematodes Paryphostomum segregatum and Schistosoma mansoni. Parasitology, 65, 223-233.

Lie K. J., Umathevy T. (1965): Studies on Echinostomatidae (Trematoda) in Malaya. X. The life history of Echinoparyphium dunni sp. n. J. Parasitol., 51, 793-799.

Lie K. J., Basch P. F., Heyneman D., Beck A. J., Ralphaudy J. (1968) : Implications for Trematode control of interspecific larval antagonism within snail hosts. Roy. Soc. Trop. Med. Hyg., 62, 299-319.

Lie K.J. (1973) : Larval Trematode antagonism: principles and possible application as a control method. Exper. Parasitol., 33, 343-349.

Lie K. J., Heyneman D., Jeyarasingham U., Mansour N., Lee H.E. (1975) : The life cycle of Echinoparyphium ralphaudyi sp. n. (Trematoda: Echinostomatidae). J. Parasitol., 61, 59-65.

Moravec F., Barus V., Rysavy B., Yousif F. (1974a) : Antagonism of Echinoparyphium recurvatum against Schistosoma haematobium in the snail Bulinus truncatus. Folia parasit. (Praha), 21, 127-141.

Moravec F., Barus V., Rysavy B., Yousif F. (1974 b) : Observations on the development of two Echinostomes, Echinoparyphium recurvatum and Echinostoma revolutum, the antagonists of human Schistosomes in Egypt. Folia Parasit. (Praha), 21, 107-126.

Nassi H. (1978) : Données sur le cycle biologique de Ribeiroia marini guadeloupensis n. sp., Trématode stérilisant Biomphalaria glabrata en Guadeloupe. Act. Trop., 35, 41-58.

Richard J., Brygoo E.-R. (1978) : Cycle évolutif du Trématode Echinostoma caproni Richard, 1964 (Echinostomatoidea). Ann. Parasitol. Hum. Comp., 53, 265-275. 
Rysavy B., Barus V., Moravec F., Yousif F. (1974): On some problems of the biological control of human Schistosomes in Egypt. Folia Parasit. (Praha), 21, 161-168.

Skrjabin K. I. (1956) : Trematodes of animals and man. Essentials of trematology (Vol. 12). Moscou (Verl. Akad. Wiss), 932 p.

Tabet-Derraz O., Belkaid M., Naceur D., Ouchefoun A. (1977): Un nouveau foyer de bilharziose urinaire en Algérie. Bull. Soc. Path. Ex., 3, 224-227.

Yamaguti S. (1971) : Synopsis of Digenetic Trematodes of Vertebrates. Keigaku publishing co., 1 and 2, 1074 p. 